\title{
The propositional approach to associative learning as an alternative for association formation models
}

\author{
JAN DE HouWer \\ Ghent University, Ghent, Belgium
}

\begin{abstract}
Associative learning effects can be defined as changes in behavior that are due to relations between events in the world. Most often, these effects are explained in terms of the formation of unqualified associations in memory. I describe an alternative theoretical explanation, according to which associative learning effects are the result of the nonautomatic generation and evaluation of propositions about relations between events. This idea is supported by many studies showing that associative learning effects are determined not only by the direct experience of events but also by prior knowledge, instructions, intervention, and deductive reasoning. Moreover, evidence supports the assumption that associative learning effects depend on nonautomatic processes. Whereas a propositional approach thus offers many new insights, questions can be raised about what the idea of association formation adds to our understanding of associative learning.
\end{abstract}

In order to survive in an ever-changing world, organisms need to detect relations between events in their environment. This statement may be a commonplace, but it does raise an important question that has intrigued many philosophers and psychologists: How do organisms manage to adapt their behavior to relations in the environment (Bouton, 2007; Hume, 1739/1987; Kant, 1781/1965)? In psychology, research on this question has been dominated by association formation models, the basic idea underlying which is that associative learning is accomplished by the formation of associations between representations in memory. An "association . . . simply connects the mental images of a pair of events in such a way that activation of one image causes activation (or inhibition) of the other" (Shanks, 2007, p. 294). Different association formation models differ in the assumptions they make about the type of representations assumed to become linked and the conditions under which associations are formed or change (e.g., Denniston, Savastano, \& Miller, 2001; Mackintosh, 1975; Pearce \& Hall, 1980; Rescorla \& Wagner, 1972; Wagner, 1985). The dominance of association formation models has been so strong that it is often not clear whether researchers use the term "associative learning" to refer to the empirical phenomenon that organisms adapt their behavior to the presence of relations in the world or to the assumed theoretical process that associations are formed between representations. In this article, I will argue that it is important to clearly distinguish between the empirical phenomenon of associative learning and the theoretical process of association formation. One of the main advantages of making that distinction is that it provides theoreti- cal freedom. It allows one to consider the possibility that associative learning as an empirical fact could be due not only to association formation processes but also to other processes.

In the first part of this article, I discuss in more detail why it is important to distinguish between the level of the empirical phenomenon or effect and the level of theoretical processes. Although this discussion is rather abstract and conceptual, it does make clear why association formation should not be seen as the only possible process that can produce associative learning effects. Hence, other theoretical approaches can and should be considered. In the second and main part of this article, I describe and evaluate one such alternative: the propositional approach. This approach leads to a host of predictions, many of which have already been confirmed empirically. In the third and final part, I discuss whether the propositional and association formation approach each offer unique insights into associative learning.

\section{PART I \\ Associative Learning As an Effect and As a Process}

Associative learning can be defined as a behavioral phenomenon: a change in the behavior of an organism resulting from changes in the relations in the world. ${ }^{1}$ In other words, associative learning is regarded as an effect, an observation attributed to a core procedure. A core procedure is an abstract regularity in the environment - that is, a regularity that can be instantiated in many different

J. De Houwer, jan.dehouwer@ugent.be 
specific procedures or situations (see De Houwer, 2007, for an in-depth discussion of the distinctions between procedure, effect, and theory). In the case of associative learning, the core procedure entails that (1) a relation between events is present in the environment and (2) the researcher examines whether the relation leads to a change in the behavior of an individual. If a change in behavior is actually observed, the change can be called an associative learning effect, once it is attributed to the relation in the environment. Associative learning as an effect is thus more than an observed change in behavior; it is an observation attributed to a certain relation. Within this framework, classical and operant conditioning can be defined as effects that differ with regard to the precise nature of the core procedure assumed to be responsible for the change in behavior. Classical conditioning refers to a change in behavior due to a relation between stimuli, whereas operant conditioning refers to a change in behavior attributed to a relation between a behavior and a stimulus (Bolles, 1979; Eelen, 1980).

Defining associative learning (be it classical or operant conditioning) as an effect has many advantages (see De Houwer, 2007). One advantage is that such a definition helps organize research. The idea that associative learning is a change in behavior resulting from a relation in the world leads to three questions. First, what does it mean to say that there is a relation in the world? This question concerns the nature of the core procedure - that is, the abstract procedural properties of how events are paired. ${ }^{2}$ The second question concerns the generality of associative learning. Research concerned with this question examines which specific implementations of the core procedure lead to associative learning effects. The third question involves the conditions that modulate the effect of the core procedure; that is, under what conditions do pairings lead to changes in behavior? Examples of modulating conditions are awareness of the CS-US relation, the availability of time and attentional resources, and the presence of knowledge, goals, and dispositions. Learning research can be organized by determining for each study how the three questions are addressed.

However, the most important advantage of defining associative learning as an effect is that it offers theoretical freedom. The fact that relations in the environment can lead to changes in behavior is the explanandum (that which needs to be explained), independent of the explanans (that which explains). In psychology, explanations are most often phrased in terms of hypothetical psychological processes. One such process is association formation. There is, however, no logical reason why association formation would be the only possible theoretical process underlying associative learning effects. At least in principle, other psychological processes could (also) be crucial. All theoretical options are open.

Despite the important advantages of defining associative learning as an effect, terms such as "associative learning" and "conditioning" are sometimes (implicitly) used to refer to the theoretical process of association formation. For instance, in many textbooks (e.g., M. Evans, Jamal, \& Foxall, 2006), classical conditioning is described as a very simple, noncognitive learning process that involves changes in involuntary responses to stimuli as the result of the contiguity-driven, unconscious formation of associations in memory. There are several risks connected to defining associative learning and conditioning in terms of a theoretical process.

First, it becomes difficult to determine whether "real" associative learning has occurred. When associative learning is defined as a theoretical process, in order to demonstrate that associative learning has occurred one does not only need to show that a change in behavior is due to a relation in the environment, but also that the impact of the relation on behavior is due to a particular theoretical process, such as association formation. The problem is that theoretical processes cannot be observed directly. For instance, nobody has ever seen an association between representations. One can observe neurons and dendrites, but a neuron is not the same as a representation and a dendrite is not the same as an association. One could infer the presence of a process indirectly on the basis of some objective, observable criterion. But this works only if that criterion is a valid indicator of the presence of the process - that is, if the process is a necessary and sufficient condition for producing the criterion. It is doubtful whether this applies to many behavioral criteria.

A second risk of defining associative learning as a process is that theoretical research is hampered thereby. First of all, because it is difficult to determine whether "real" associative learning has occurred, it also becomes difficult to study the phenomenon. Second, part of the answer about the theoretical processes underlying associative learning effects is already postulated by definition. Because of this, other theoretical possibilities are not even considered, and the insights that this other viewpoint might offer remain hidden.

On the basis of the arguments presented above, I define associative learning as an effect rather than as a theoretical process. Within this conceptual framework, it is obvious that a thorough study of the empirical phenomenon of associative learning (i.e., associative learning as an effect) can be accomplished only if one allows for the possibility that various types of theoretical processes might underlie this phenomenon. In the next section, I will argue that propositional processes are an important source of associative learning effects.

\section{PART II A Propositional Approach to Associative Learning}

In recent years, several models of associative learning have been put forward that adopt a propositional approach. These include causal model theory (Waldmann, 2000; Waldmann \& Holyoak, 1992) and the higher order or propositional reasoning account (De Houwer, Beckers, \& Vandorpe, 2005; Lovibond, 2003). All propositional models share two core assumptions. First, associative learning effects are assumed to depend on the formation and evaluation of propositions. Second, nonautomatic processes are assumed to intervene in the formation and evaluation of 
these propositions. In the following paragraphs, I will discuss in detail the nature and implications of the two core assumptions of propositional reasoning models, as well as the empirical evidence supporting these assumptions.

\section{First Core Assumption: Associative Learning Effects Depend on the Formation and Evaluation of Propositions}

Propositions differ in important ways from associations. Most prominently, propositions but not associations imply a truth value (e.g., Strack \& Deutsch, 2004). Propositions are statements about a state of affairs in the world and can therefore differ in the extent to which they are accurate (or are believed to be). Associations, on the other hand, are simply unqualified links between representations through which activation can spread (e.g., Dickinson, 1980, p. 85; Shanks, 2007, p. 294). The existence of an association or the spread of activation is a state of affairs rather than a statement about a state of affairs. Hence, associations are neither true nor untrue, they just are.

Because propositions are statements about a state of affairs, they can refer not only to the presence of a relation between events but also to the manner in which events are related. Consider the propositions "cue A is a cause of outcome O" and "cue A is an effect of the outcome." Both imply that $\mathrm{A}$ and $\mathrm{O}$ are related but they differ with regard to the way in which $\mathrm{A}$ and $\mathrm{O}$ are assumed to be related. In more abstract terms, propositions can specify not only the strength of relations in the world but also their structure (see Lagnado, Waldmann, Hagmayer, \& Sloman, 2007, for an excellent discussion of this point in the context of causal learning). It is essential that organisms try to discover the structure of relations in the world; otherwise, they will not be able to respond differently on the basis of relations equivalent in strength but different in structure. As an example, assume that a covariation is observed between certain substances in the blood and a certain disease (see Waldmann \& Holyoak, 1992). A blood transfusion would make little sense as a cure for a disease if the substances in the blood were a harmless effect of the disease; but it would make sense if the substances in the blood were the cause of the disease. Whereas propositions can capture the difference between structure and strength, associations are sensitive only to the strength of relations (Lagnado et al., 2007). In association formation models, the links between representations are unqualified; that is, they do not include information about how the events are related (e.g., Shanks, 2007, p. 294).

Propositional models postulate that associative learning effects will depend on the truth evaluation of the propositions considered to be relevant to the behavior being measured. In other words, whether and in which way relations between events influence behavior (i.e., whether and which associative learning effects will occur) depends on the extent to which statements about those relations (i.e., propositions) are considered to be true. Whether a proposition is considered to be true depends on the extent to which it is consistent with other propositions believed to be true (e.g., Gawronski \& Strack, 2004). Importantly, these other propositions can originate from a wide vari- ety of sources, including prior knowledge, experience, instructions, deductive reasoning, and intervention. Because associative learning effects are assumed to reflect the truth evaluation of propositions, each factor that determines the truth evaluation of propositions should also determine associative learning effects. In the following paragraphs, I will present some of the evidence for the effects of propositions based on prior knowledge, experience, instructions, intervention, and deductive reasoning.

Many of the findings in support of propositional models originated from studies on blocking in human contingency learning. In studies on human contingency learning (see De Houwer \& Beckers, 2002b, for a review), participants see a series of trials on which certain cues and outcomes are either present or absent. On the basis of the observed trials, participants afterward have to judge the relation between certain cues and certain outcomes. Human contingency learning effects are structurally similar to classical conditioning effects. In both cases, changes in behavior (contingency judgments or conditioned responses [CRs]) result from the pairing of events (cues and outcomes or conditioned stimuli [CSs] and unconditioned stimuli [USs]). Similar phenomena can be observed in both types of learning. One of the most important of these phenomena is blocking, which refers to the observation that a CS X will not evoke a CR after it is paired with a US, provided that it is always presented together with a second CS A previously paired with the US (i.e., A+ trials followed by $\mathrm{AX}+$ trials, where the letters stand for the presence of cues and + stands for the presence of the US or outcome; Kamin, 1969). In human contingency learning studies, this means that ratings for the strength of the relation between $\mathrm{X}$ and the outcome will be lower when $\mathrm{AX}+$ trials are combined with $\mathrm{A}+$ trials, compared with when only $\mathrm{AX}+$ trials are presented. As will become apparent from the following paragraphs, the study of blocking effects has provided strong evidence for the involvement of prior knowledge, experience, instructions, intervention, and deductive reasoning in associative learning.

Propositions based on prior knowledge. Studies on blocking in human contingency learning have shown that prior knowledge does have an important impact on associative learning effects. Most importantly, Waldmann (2000; Waldmann \& Holyoak, 1992) showed that blocking effects are more outspoken if the cues $\mathrm{A}$ and $\mathrm{X}$ are described as potential causes of the outcome (e.g., substances in the blood that could cause a disease) than when $\mathrm{A}$ and $\mathrm{X}$ are said to be potential effects of the outcome (e.g., substances in the blood that result from a disease; see De Houwer, Beckers, \& Glautier, 2002, for related evidence). Once cues have been categorized as potential causes of the outcome, participants can fall back on their prior propositional knowledge about causal relations, including the general knowledge that causes tend to have additive effects. The observation that the outcome is identical on $\mathrm{AX}+$ trials and $\mathrm{A}+$ trials shows that $\mathrm{X}$ does not add anything to the effect of A. This observation, combined with the prior knowledge that causes typically have additive effects, is inconsistent with the proposition " $\mathrm{X}$ is a cause of the outcome." If we assume the observation and 
the additivity assumption to be correct, the proposition " $\mathrm{X}$ is a cause of the outcome" must be incorrect. On the other hand, when $\mathrm{A}$ and $\mathrm{X}$ are considered to be effects of the outcome, information about the $\mathrm{A}$-outcome relation is not relevant to assessing the truth of the proposition " $\mathrm{X}$ is an effect of the outcome." The extent to which X is an effect is independent of the extent to which A is an effect of the outcome. Hence, blocking effects should be less likely to occur when the cues are potential effects of the outcome than when they are potential causes of the outcome. This prediction has been verified in several studies (see Lopez, Cobos, \& Caño, 2005, for a review).

If there is an inconsistency between a to-be-evaluated target proposition (e.g., "X is a cause of the outcome"), propositional knowledge about observed events (e.g., "X does not add anything to the effect of A"), and prior propositional knowledge (e.g., "Causes have additive effect"), the inconsistency can be solved not only by evaluating the target proposition as being false but also by questioning the validity of the observed events or prior knowledge. Questioning the validity of prior knowledge is likely, especially when there are indications that this knowledge is not valid in the current context; again, there is strong evidence to support this argument. First, De Houwer et al. (2002) informed participants assigned to a maximal condition that the outcome always occurred with an intensity of 10 on a scale of 10 , both when only Cue A was present $(\mathrm{A}+)$ and when both $\mathrm{A}$ and $\mathrm{X}$ were present $(\mathrm{AX}+)$. In this condition, $\mathrm{A}$ on its own causes the outcome to a maximal extent and, because of ceiling effects, participants cannot verify whether $\mathrm{X}$ adds anything to the effect of A. The additivity assumption is not valid when ceiling effects are possible. Hence, the proposition " $\mathrm{X}$ is a cause of the outcome" cannot be evaluated on the basis of the prior knowledge that causes tend to have additive effects and the observation that the outcome is the same on $\mathrm{AX}+$ and $\mathrm{A}+$ trials. In a second, submaximal condition, participants saw the exact same events, except that the outcome was said to always occur with a submaximal intensity of 10 on a scale of 20 . These participants could verify that $X$ did not add anything to the effect of $A$ and thus that $X$ was not a cause of the outcome. In line with these arguments, blocking was significantly stronger in the submaximal than in the maximal condition.

A second way to question the validity of the additivity assumption is to simply tell participants that it is not valid. For instance, in a human electrodermal conditioning preparation (i.e., USs are aversive shocks and changes in skin conductance are the CR), Mitchell and Lovibond (2002) informed participants in the additive condition that two colors (CSs) that separately each led to an electric shock (US) would lead to a double electric shock when presented together. This information supports the additivity assumption. Participants in the nonadditive condition were told that two colors that individually led to an electric shock would be followed by the same shock when presented together: This information contradicts the additivity assumption. Supporting the idea that blocking is the result of inferences based on the additivity assumption, blocking was significantly stronger in the additive condi- tion than in the nonadditive condition. Propositions can be questioned not only by instructions but also by experience. For instance, Beckers, De Houwer, Pineño, and Miller (2005) exposed their participants to a training phase that consisted of events that either confirmed or contradicted the assumption that causes have additive effects. Again, blocking was reduced in the condition where the additivity assumption was invalid. In sum, these studies show that participants do take into account prior knowledge about the additivity of causes, but only when this knowledge is thought to be valid.

The additivity assumption cannot only be put aside if there is evidence to doubt its validity; it can also be replaced by new propositional assumptions about how the effects of cues or causes interact. Consider the well-known studies of Shanks and Darby (1998). They presented A+, $\mathrm{B}+, \mathrm{AB}-, \mathrm{C}-, \mathrm{D}-$, and $\mathrm{CD}+$ trials together with $\mathrm{I}+$, $\mathrm{J}+, \mathrm{M}-$, and $\mathrm{N}-$ trials. During a test phase, participants judged that the outcome was more likely to occur after the (previously unseen) compound MN than after the (also previously unseen) compound IJ. Judgments thus reflected the newly learned propositional rule that the likelihood of the outcome after a compound of two stimuli (i.e., $\mathrm{AB}-$, $\mathrm{CD}+)$ is the reverse of the likelihood of the outcome after the elements of the compound (i.e., $\mathrm{A}+, \mathrm{B}+, \mathrm{C}-, \mathrm{D}-$ ). New assumptions can be based not only on experience but also on instructions. For instance, Mitchell, Killedar, and Lovibond (2005) instructed participants that whatever was true for one stimulus was also true for the other stimuli with which it was paired. Therefore, after observing AX+ trials and $\mathrm{A}+$ trials, they should infer that $\mathrm{X}$ is actually a cause of the outcome. Mitchell et al. indeed observed such a reversed blocking effect under these conditions.

The additivity assumption is an example of abstract prior knowledge, which, because of its abstract nature, can influence learning in a wide variety of situations with a wide variety of stimuli. It should be clear, however, that people also possess more specific prior knowledge about particular stimuli and events. For instance, studies have confirmed that a stimulus is more likely to be seen as a cause of an event if participants have prior knowledge about a mechanism by which the stimulus can cause the event (Bullock, Gelman, \& Baillargeon, 1982; Lien \& Cheng, 2000). Also, new evidence is interpreted in the light of old evidence for a certain relation (e.g., Maldonado, Catena, Perales, \& Cándido, 2007); hence, both abstract and specific prior knowledge determine associative learning in a way consistent with propositional models.

Propositions based on experience. The fact that experience can drive associative learning effects is, of course, uncontroversial. What is unique about propositional models is that experience is assumed to lead to associative learning effects only via propositional beliefs about the experienced events. In other words, what matters is not so much the objective events themselves, but subjective beliefs about events - that is, the extent to which propositions about the events are believed to be true. We have already seen that learning effects depend on whether stimuli are believed to be causes or effects (e.g., Waldmann \& Holyoak, 1992). Note that this illustrates the general principle that learning 
depends on the mental categorization of events. Once an event is categorized and interpreted in a certain manner (e.g., as a cause), the propositional knowledge inherent to the chosen category (e.g., causes have additive effects) can be used to determine how events are related.

Not only beliefs about the nature of experienced events are important; so are beliefs about the actual presence or absence of events. For instance, De Houwer (2002) showed $\mathrm{A}+$ trials followed by $\mathrm{AX}+$ trials, but obscured the position where $\mathrm{X}$ could appear during the $\mathrm{A}+$ trials. Hence, participants could not be sure about whether $X$ was present or absent on the $\mathrm{A}+$ trials. Those participants who said that they believed that $X$ was absent on the $\mathrm{A}+$ trials showed a large blocking effect, because they could verify that $\mathrm{X}$ did not add anything to the effect of A, whereas those who believed that $\mathrm{X}$ was present on the $\mathrm{A}+$ trials did not show a blocking effect, because they thought they only observed the joined effect of both causes. In a second experiment, De Houwer (2002) manipulated the beliefs about the presence of $\mathrm{X}$ on the A+ trials by telling participants at the end of the learning phase that $\mathrm{X}$ was either present or absent. Only the participants who were told that $\mathrm{X}$ was absent on the $\mathrm{A}+$ trials showed a blocking effect. These experiments show that associative learning effects depend more on the extent to which propositions about the presence of events are believed to be true than merely on the objective events themselves (see Waldmann, 2000, Experiment 2, for related results).

Another way to manipulate propositional beliefs about the presence of events is to influence whether participants consciously remember certain events from the past. Studies on backward blocking suggest that associative learning effects indeed depend on what is remembered about the actual events. There is ample evidence showing that blocking can be observed when AX+ trials are presented before A+ trials. However, such backward blocking effects depend on whether participants remember the AX+ trials at the time that the $\mathrm{A}+$ trials are presented. For instance, it has been demonstrated that the magnitude of backward blocking is correlated with the accuracy of memory for the AX+ trials (e.g., Melchers, Lachnit, \& Shanks, 2004; Vandorpe, De Houwer, \& Beckers, 2007). Likewise, manipulations that disrupt memory for the AX+ trials lead to a reduction in backward blocking (e.g., Aitken, Larkin, \& Dickinson, 2001; Dickinson \& Burke, 1996). Propositional models provide a straightforward explanation for these findings. Provided that there are no ceiling effects, seeing $\mathrm{A}+$ trials after $\mathrm{AX}+$ trials allows one to conclude that $\mathrm{X}$ is not a cause of the outcome because $\mathrm{X}$ does not add anything to the effect of A. However, if participants do not remember the $\mathrm{AX}+$ trials while seeing the $\mathrm{A}+$ trials, they do not have all the propositional knowledge necessary to infer that the $\mathrm{X}$ is not a cause of the outcome. Forward blocking, on the other hand, should not depend on memory for the AX+ trials. In a forward blocking design, $\mathrm{A}+$ trials are presented before $\mathrm{AX}+$ trials. Hence, participants have all the crucial information for inferring the status of $\mathrm{X}$ at the time that they observe the $\mathrm{AX}+$ trials. They do not need to remember the AX+trials, because the latter are presented at the time the inference about $\mathrm{X}$ can be made; all that is needed is recollection of the A+ trials. Studies indeed show that forward blocking does not depend on memory for the AX+ trials (e.g., Dickinson \& Burke, 1996; Melchers et al., 2004) unless participants are prevented from making the inference about $\mathrm{X}$ during the AX+ trials (Vandorpe et al., 2007). Although some associative models can explain the fact that backward but not forward blocking depends on memory for the AX+ trials (Dickinson \& Burke, 1996; Melchers et al., 2004; Van Hamme \& Wasserman, 1994), none of the associative models is compatible with the fact that forward blocking can depend on memory for the AX+ trials when participants are prevented from making inferences during the $\mathrm{AX}+$ trials. The latter finding provides unique support for propositional models.

Experience can result not only in beliefs about the presence or nature of specific events or relations, it can also change more abstract propositional beliefs. For instance, the studies of Beckers et al. (2005; also see Lovibond, Been, Mitchell, Bouton, \& Frohardt, 2003) mentioned above showed that presenting events that confirm or contradict the additivity assumption influences blocking effects. Experiencing those events thus appears to lead to changes in the extent to which the (abstract) additivity assumption is believed to be true. Likewise, the results of Shanks and Darby (1998) show that new abstract knowledge can be learned on the basis of experience. As described above, participants learned the rule that the likelihood of the outcome after a compound of two stimuli (i.e., $\mathrm{AB}-, \mathrm{CD}+$ ) is the reverse of the likelihood of the outcome after the elements of the compound (i.e., $\mathrm{A}+$, $\mathrm{B}+, \mathrm{C}-, \mathrm{D}-$ ). Regardless of whether propositions are specific (i.e., apply to one particular relation; e.g., "A is followed by the outcome") or abstract (i.e., apply to many relations; e.g., "causes are additive"), experienced events seem to influence learning only by their impact on the truth evaluation of propositions.

Propositions based on instructions. The studies of De Houwer (2002, Experiment 2) that I discussed in the previous section already show that associative learning effects can be influenced by instructions about ambiguous past experiences. Other evidence demonstrates also that instructions about events that have not been experienced can influence associative learning effects; for instance, if one informs a participant that a tone will always be followed by a shock, that tone will thereafter evoke a conditioned galvanic skin response (GSR), even though tone and shock have never actually been presented together (e.g., Cook \& Harris, 1937). Likewise, if one first presents tone-shock trials, and then informs the participants that the tone will no longer be followed by the shock, the conditioned GSR will be dramatically reduced (e.g., Colgan, 1970). The latter result demonstrates that verbal instructions about a relation can lead to the same effects as the actual experience of a relation can, and also suggests that propositional beliefs derived from actual experience can interact with propositional beliefs derived from verbal instructions.

Recent studies showed that these conclusions also hold for more complex learning phenomena. Lovibond (2003) 
found that presenting $\mathrm{A}-$ trials ( $\mathrm{A}$ is presented without the US) after AT + trials resulted in an increase in the conditioned GSR toward T. The exact same result was found when the AT + and A - were described verbally rather than actually presented (Experiment 2), or when the AT+ trials were actually presented but the subsequent $\mathrm{A}$ - trials were replaced by the verbal message that $\mathrm{A}$ was a safe cue that would not be followed by the US (Experiment 3 ).

As was the case for experienced events, instructions can influence abstract propositional knowledge also. For instance, telling participants that causes do or do not have additive effects is enough to influence the size of blocking effects (Mitchell \& Lovibond, 2002). Likewise, simply telling people about a new rule (e.g., "whatever is true for one stimulus is also true for the other stimuli with which it was paired") leads to behavior consistent with that rule (Mitchell et al., 2005).

Propositions based on intervention. Philosophers have long recognized that intervention offers a privileged route to the discovery of relations in the world (e.g., Bacon, 1620/1994; see Lagnado et al., 2007). More recent research confirms that, compared with mere observation, intervention does help participants to uncover the way in which events are related (e.g., Steyvers, Tenenbaum, Wagenmakers, \& Blum, 2003). People also seem to be aware of the fact that intervention and observation can have different implications. Take the study of Waldmann and Hagmayer (2005), in which one group of participants was told that the (fictitious) hormones "sonin" and "xanthan" were both effects of the hormone "pixin." On the basis of this information, they correctly predicted that "xanthan" was likely to be present when "sonin" was observed. In this case, the presence of "sonin" is indicative of the presence of "pixin," which is also the cause of "xanthan." When the participants learned that "sonin" was present because of an intervention (i.e., an injection of "sonin" in the blood), they did not believe that the presence of "xanthan" was likely. This is because the intervention renders "sonin" independent of its usual cause, "pixin," meaning that the presence of "sonin" can no longer be used as an indicator of the presence of "pixin." Hence, participants realized that merely observing an event, or intervening to cause an event, are not the same thing.

Waldmann and Hagmayer (2005) also tested a second group of participants who were told that the hormone "sonin" was a cause of the hormone "pixin," and that "pixin" in its turn produced the hormone "xanthan." On the basis of this information, the presence of "sonin" should be accompanied by the presence of "xanthan," whether the presence of "sonin" was merely observed or caused by an intervention. This second group of participants indeed made the same predictions in the observation as in the intervention condition. The fact that participants reacted differently, according to whether "sonin" was said to be an effect of "pixin" (first group of participants) or a cause of "pixin" (second group of participants), supports the idea that people use propositional information to form hypotheses about the presence of events (i.e., whether "xanthan" is present). For all participants, "sonin" was related to "pixin"; only the nature of the relation differed.
Participants must therefore have represented and taken into account the nature of the relation. Because this can be achieved on the basis of propositions, but not of associative links between representations, the results of Waldmann and Hagmayer provide strong support for propositional models and against association formation models. It must be said, though, that these studies deal primarily with the effect of propositional knowledge on predictions about the effects of interventions, rather than with how interventions can lead to the discovery of relations in the world. Few studies have looked at the latter issue (but see Steyvers et al., 2003).

Propositions based on deductive reasoning. The evidence presented above already shows that reasoning is crucial in associative learning. It is the mechanism that allows one to assess the truth of a proposition by examining the extent to which the proposition is consistent with other propositions believed to be true. But deductive reasoning can also provide the input for the truth evaluation mechanism; that is, the truth evaluation of one proposition can form the basis of the truth evaluation of another proposition. This idea is best illustrated by a phenomenon known as higher order retrospective revaluation. De Houwer and Beckers (2002a, 2002c) first presented AB + trials, then $\mathrm{AX}+$ trials, and finally either $\mathrm{B}-$ or $\mathrm{B}+$ trials. Whether $\mathrm{B}+$ or $\mathrm{B}-$ trials were presented influenced not only the ratings for the $\mathrm{A}$-outcome relation but also the ratings for the $\mathrm{X}$-outcome relation. At first sight, participants cannot arrive at a valid conclusion about the causal status of $\mathrm{X}$, because no trials are presented in which $\mathrm{A}$ is the only cue. When, however, participants see $\mathrm{AB}+$ and $\mathrm{B}-$ trials, they can infer that $\mathrm{A}$ is a cause of the outcome because the outcome occurs on the $\mathrm{AB}+$ trials, despite the fact that $\mathrm{B}$ on its own does not cause the outcome. Given this inferred propositional knowledge, they can subsequently infer that $\mathrm{X}$ is not a cause of the outcome because the observed effect of A and X together is the same as the hypothesized effect of $\mathrm{A}$ alone. If $\mathrm{B}+$ trials are presented rather than $\mathrm{B}$ - trials, participants can conclude that $\mathrm{A}$ is not a cause of the outcome (because B alone has the same effect as A and B together), and that X is therefore a cause of the outcome (because the outcome is thought to be more likely when $\mathrm{A}$ and $\mathrm{T}$ are presented together than when $A$ is presented on its own).

Which propositions are truth evaluated? Until now, I have focused on the question of how the truth of propositions is evaluated. I have seen that this can be done by examining the extent to which the to-be-evaluated target proposition is consistent with other propositions that originate from prior knowledge, experience, instruction, intervention, and deductive reasoning. This leaves open the question of which propositions are truth evaluated and determine behavior; in order to answer this, it is important to realize that any single relation in the world can be described by many different propositions. This is because a relation between events is a multifeatured object. One important feature is the degree of statistical contingency between the related events. This expresses the extent to which the probability of one event depends on the presence of the other event [i.e., $P(\mathrm{E} 1 / \mathrm{E} 2)$ compared with 
$P(\mathrm{E} 1 / \sim \mathrm{E} 2)]$. But there are also other features. The causal nature of a relationship, for instance, depends on the assumed presence of a causal power or mechanism by which one event can bring about another event (e.g., Cheng, 1997). One can also describe the conditions under which a statistical contingency is observed; for instance, whether it can be observed when the presence of a third event is kept constant [e.g., $P(\mathrm{E} 1 / \mathrm{E} 2 . \mathrm{E} 3)$ vs. $P(\mathrm{E} 1 / \sim \mathrm{E} 2 . \mathrm{E} 3)]$. Another way to describe a relation between events is in terms of the conditional probability of one event given the presence of another event [i.e., $P(\mathrm{E} 1 / \mathrm{E} 2)]$. An even more basic feature of a relation is the degree of contiguity between events. This simply refers to the number of times that two events go together.

Each of these features can be described by a proposition and each of these propositions can be truth evaluated. Whether and in what way behavior is affected by a relation between events depends on the truth evaluation of the proposition that underlies that particular behavior. From this perspective, therefore, a major question in the study of associative learning is the question of which propositions underlie which type of behavior. In some cases, the answer to this question is straightforward. For instance, one can simply ask participants to judge a particular feature of a relation between events; that is, one can directly assess the degree to which participants believe that a certain proposition is true. Because each proposition describes different aspects of the same relation, the different judgments should depend on different properties of that relation. Matute and colleagues (e.g., Matute, Arcediano, \& Miller, 1996; Vadillo \& Matute, 2007; Vadillo, Miller, \& Matute, 2005) reported evidence that supports this prediction. For instance, Matute et al. showed that blocking-like effects do occur when participants make causal judgments (i.e., the extent to which one event is seen as a cause of a second event), and predictive-value judgments (i.e., the extent to which one event is seen as a reliable predictor of the second event), but not when they judge the extent to which two events co-occurred. Likewise, Vadillo et al. demonstrated that the probability of one event in the absence of another event influences causal judgments and predictive-value judgments (see above) but not prediction judgments (i.e., how likely it is that one event will occur given the presence of a second event).

When participants are not directly asked to judge a particular feature of the relation between events, it is less clear what proposition will determine the behavior. Nevertheless, predictions can be made on the basis of normative arguments - that is, on the basis of what would be most rational or adaptive. Take the case of preparatory responses - responses aimed at dealing with an anticipated event. De Houwer, Vandorpe, and Beckers (2007) pointed out that, from a normative perspective, such behaviors should depend on propositions about the probability of the anticipated event given the current situation. For instance, if a certain cue is present in a certain context, the decision to prepare for an event should depend on propositions about how likely the event is when the cue is present in that context. Given the assumption that the presence of causes does not change over time, the best way to esti- mate the likelihood of an event in a certain situation is to estimate the likelihood of that event in previous, identical situations. Hence, decisions about whether to prepare for an event when a certain cue is present should depend on the probability of that event in previous situations where the cue was present [i.e., $P(\mathrm{E} / \mathrm{C})]$, but not on the probability of the event in previous situations where the cue was absent [i.e., $P(\mathrm{E} / \sim \mathrm{C})]$. The results of De Houwer et al. (2007) confirmed that preparation responses depended only on the former probability $[P(\mathrm{E} / \mathrm{C})]$.

Although a priori arguments can be constructed about which behaviors should be determined by which propositions, ultimately this question can be solved only empirically. Several research strategies can be followed in this context. Given a hypothesis about the propositions that underlie a certain learned behavior, one can examine whether the experiences that should affect the truth evaluation of that proposition indeed do affect the learned behavior. Assume that, like De Houwer et al. (2007), one can show that an associative learning effect depends on the probability of an event in the presence of a cue $[P(\mathrm{E} / \mathrm{C})]$ but not on the probability of the event in the absence of the cue $[P(\mathrm{E} / \sim \mathrm{C})]$. This would support the conclusion that the observed effect depends on propositions about conditional probabilities or contiguity rather than about the statistical contingency or causal relation between events (see De Houwer et al., 2007). To determine whether an effect depends on propositions about the statistical contingency or propositions about the causal relation between events, one could examine whether and how people are likely to intervene in the presence of one event in order to control the presence of the other event (e.g., Waldmann \& Hagmayer, 2005). Regardless of the usefulness of these particular research strategies, the fact remains that, from a propositional point of view, different associative learning effects (i.e., associatively induced changes in different types of behavior) could be determined by different aspects of the procedure (i.e., different aspects of the relation between events). Effects involving one type of behavior could, for instance, depend on the extent to which two events co-occur, but not on the extent to which they occur separately. Effects involving other types of behavior might be influenced by both the extent to which events co-occur and occur separately. Such a diversity within associative learning effects follows naturally from the idea that different associative learning effects can depend on the truth evaluation of different propositions.

What factors determine the generation of propositions? Until now, the emphasis has been on the factors that influence the truth evaluation of propositions. But before the truth of a proposition can be evaluated, it first needs to be generated. According to propositional models, the same factors that influence the truth evaluation of propositions also determine the generation of these propositions.

First, hypotheses about relations between events can be generated on the basis of prior knowledge. For instance, when looking for the causes of an event, people most often first consider those stimuli for which there is a plausible mechanism by which the stimulus can cause the event 
(e.g., Bullock et al., 1982; Lagnado et al., 2007). The idea that prior knowledge about causal mechanisms has an important impact on the generation of propositions about relations in the world is in line with the highly selective character of associative learning (e.g., Garcia \& Koelling, 1966). It indeed seems to be the case that humans and animals learn a relation more easily if there is a plausible causal mechanism linking the related events (e.g., Testa, 1974). Organisms also take into account very specific knowledge about prior experiences. For instance, once a relation between two events has been discovered in the past, it is likely that this knowledge is used to generate propositions about similar events in the present.

Second, organisms are capable of detecting relations between events purely on the basis of the experienced presence or absence of these events, although experience clearly has its limitations as a source of learning. In the real world, a large number of events occur at any given time. Moreover, related events are often separated by time, so past events also need to be considered. Hence, organisms are confronted with an almost infinite number of possible relations. ${ }^{3}$ For every cause or predictor of a particularly important event, there are so many other potential causes or predictors that it would be difficult, or even impossible, for an organism to detect one particular (causal) relation without having some kind of clue that allows them to limit the number of possible relations that need to be considered. Most association formation models do allow for selectivity in learning by taking into account the salience of or attention allocated to stimuli (e.g., Mackintosh, 1975; Pearce \& Hall, 1980; Rescorla \& Wagner, 1972). Given the computational complexity involved in detecting relations between events, it would be surprising if organisms did not also take into account factors such as abstract and concrete prior knowledge (e.g., Lagnado et al., 2007). A second limitation of experience as a source of learning is that the experienced presence or absence of events provides information primarily about the strength of a relation between events but not about the structure of this relation (e.g., Lagnado et al., 2007). As noted earlier in this article, the strength of a relation refers to the statistical contingency - that is, whether, and to what extent, two events are related. The structure of a relation qualifies how the events are related (e.g., in a causal or merely predictive fashion). It has been argued convincingly that, except in rare cases, statistical contingency provides insufficient information to infer whether a relation is causal (e.g., Pearl, 2000). The presence of statistical contingency between events can be a clue for generating hypotheses about causal relations, but it is not a perfect clue. Other information needs to be taken into account.

A third factor that can be used to generate propositions about relations between events are instructions. Instructions can point to possible relations either indirectly (e.g., by increasing the salience of certain events) or directly (i.e., by simply providing a proposition about a possible relation). Note that in many experiments with humans, instructions directly specify a proposition and participants are asked only to indicate the extent to which they believe that the proposition is true (e.g., "Is A related to the outcome?"). In these cases, participants do not need to generate propositions. Their only task is to evaluate the truth of the propositions provided by the experimenter.

Finally, propositions about relations in the world can result from deductive reasoning. For instance, professional scientists often derive ideas about possible relations in the world from theories. However, there is little research about the extent to which people use deductive reasoning in daily life as a means of generating hypotheses about possible relations in the world. More generally, associative learning research has focused more on how propositions are truth evaluated than on how they are generated. In order to disentangle the two topics, procedures are needed that assess not only which propositions are ultimately judged to be true, but also which ones have been previously entertained and rejected. Concurrent think-aloud protocols could provide a useful tool in this context (e.g., Ericsson \& Simon, 1993; Lucas \& Ball, 2005).

\section{Second Core Assumption: Nonautomatic Processes Intervene in the Generation and Truth Evaluation of Propositions}

As argued earlier, propositional models are built upon two core assumptions: (1) Associative learning effects depend on the generation and truth evaluation of propositions and (2) nonautomatic processes intervene in the generation and truth evaluation of propositions. Until now, the focus has been on the first assumption and the evidence supporting it. An analysis of this assumption led to many new insights into the factors taken into account when generating and evaluating propositions, and thus when learning about relations in the world. However, the first assumption says little about the manner in which these factors are taken into account when generating and evaluating propositions. The second core assumption does tell more about how propositions are generated and evaluated; it is assumed to happen in a nonautomatic manner. This raises two questions. First, what does it mean to say that a process is nonautomatic? Second, is there evidence to support the claim that the processes underlying associative learning are nonautomatic?

The answer to the first question is based on the featurebased view of automaticity (e.g, Bargh, 1992; Moors \& De Houwer, 2006). To say that a process is automatic or nonautomatic tells something about the conditions under which the process can operate. One historically important view on automaticity is that there are two sets of mutually exclusive processes: automatic processes and nonautomatic processes. According to this all-or-none view of automaticity, all nonautomatic processes have the same features (i.e., they occur only when certain conditions are fulfilled), whereas all automatic processes have the opposite features (i.e., they can occur when those conditions are not fulfilled). It has become clear, however, that the all-or-none view is incorrect. Studies have demonstrated that most processes possess both features typical of nonautomatic processes and those typical of automatic processes (e.g., Bargh, 1992). An important implication of this conclusion is that one cannot simply characterize a 
process as automatic or nonautomatic. Rather, it is necessary to always specify the sense in which a process is automatic - that is, to specify which automaticity features it possesses and which it does not. In line with these ideas, a nonautomatic process can be described as a process that operates only if the organism (1) is aware of certain aspects of the process, its input, or its output, (2) has sufficient cognitive resources, (3) is given sufficient time to initiate and complete the process, or (4) has certain goals related to the process or other processes. As indicated by the conjunction "or," these different conditions or features are not assumed to always co-occur.

In what way are the processes responsible for the generation and evaluation of propositions nonautomatic? Proponents of propositional models describe these processes as a form of conscious, effortful, time-consuming, and controlled reasoning (e.g., De Houwer, Beckers, \& Vandorpe, 2005). Whereas it remains unclear how reasoning is actually achieved, such statements do clarify that reasoning is thought to be nonautomatic in that it depends on (1) awareness; (2) cognitive resources; (3) time; and (4) goals. These assumptions are crucial, in that they point to the conditions necessary for learning effects to occur. If reasoning is nonautomatic with regard to these features, and if associative learning effects depend on reasoning, associative learning effects should be observed only under the conditions that allow for reasoning to occur. In the following paragraphs, I will present a brief overview of the evidence concerning this prediction.

Awareness. The feature "awareness" does not refer to one feature but to a set of different features regarding the operation of a process (e.g., Bargh, 1992; Moors \& De Houwer, 2006), a fact that is often overlooked. Feature "awareness" can refer to awareness of (1) the input of the process; (2) the operation of the process itself; (3) the output of the process; or (4) the effect of the process on behavior. Most, if not all, studies on associative learning and awareness have focused on the link between associative learning effects and contingency awareness - that is, awareness of the relation between events. From the perspective of propositional models, knowledge about the relation between events is propositional knowledge. Hence, research on learning and contingency awareness deals with the question of whether learning effects depend on awareness of the output of the process responsible for the generation and evaluation of propositions (i.e., awareness of propositions about relations). ${ }^{4}$ Assuming that the truth evaluation of a proposition can take place only if one is aware of the proposition, one can predict on the basis of propositional models that there will be a close relation between contingency awareness and associative learning. Although it is beyond the scope of this article to present a review of the literature, it seems safe to conclude that there is a lot of evidence to support this prediction and very little, if any, strong evidence for associative learning without contingency awareness (see Lovibond \& Shanks, 2002, for a review).

Interestingly, there is much less research about whether associative learning depends on awareness in other ways. There are several studies on learning with subliminally presented stimuli. One could regard these as related to the question of whether learning depends on awareness of the input of the processes responsible for the formation and evaluation of propositions. Assuming that one needs to be aware of the separate events (i.e., the input) before one can form propositional knowledge of the relation between those events (i.e., the output), propositional models lead to the prediction that associative learning effects should depend on awareness of the events being related. To support this prediction, there is little evidence for associative learning in the absence of awareness of the related events (see Lovibond \& Shanks, 2002, for a review).

It is less clear whether associative learning effects depend on, or are assumed to depend on, awareness of the process by which propositions are formed. Intuitively, hypotheses about possible relations in the world often seem to pop up without awareness of how the proposition was formed. Also, sometimes propositions just "feel" right, even if we cannot explain why. Such experiences are not inconsistent with a propositional view, if one assumes that past experiences can influence current behavior and thinking in an unconscious, automatic manner, as the result of the activation of memory traces. As will be discussed later on, such automatic effects of memory do not necessarily imply the operation of an association formation process. In the present context, however, the important point is that claims about the relation between learning and awareness are not necessarily the same for all aspects of the relation between learning and awareness. Although researchers are rarely explicit about this, one could postulate that learning depends on awareness of the relation (output) and the events to which one is exposed (input), but not on the process by which being exposed to the events leads to a proposition about the relation between the events.

To the best of my knowledge, there is very little research about whether learning does depend on awareness of the process by which propositions are formed and evaluated. One potentially relevant study was reported by Vandorpe, De Houwer, and Beckers (2005). They replicated earlier studies showing that forward blocking (i.e., the relation between $\mathrm{X}$ and US is judged to be weaker when $\mathrm{AX}+$ trials are preceded by $\mathrm{A}+$ trials) depends on ceiling effects (e.g., De Houwer et al., 2002) and secondary task load (De Houwer \& Beckers, 2003). The new aspect of their study was that they asked participants to report verbally how they arrived at their judgments about the relation between events. In line with the idea that blocking is due to the conscious inference that $\mathrm{X}$ cannot be a cause of the outcome if it does not add anything to the effect of A, only participants who showed a blocking effect reported this blocking inference as a justification for their ratings of X. Moreover, variables that influenced blocking also influenced the number of people who reported a blocking inference. Finally, participants who reported a blocking inference under conditions where it was not valid (i.e., when A on its own produced the maximal effect) did show a blocking effect but were more uncertain about their causal ratings for $\mathrm{X}$ than were participants who reported a blocking inference and could be certain that the inference was valid (i.e., when the ef- 
fect of A was submaximal). It is important to note that the confidence ratings were collected at the same time as the causal ratings were, and that both were collected before the verbal reports. Therefore, the pattern of results observed in the confidence ratings strongly supports the idea that the verbal reports were not an ex post facto justification of their ratings, but actually reflected the arguments that participants used to arrive at their causal ratings. The studies of Vandorpe et al. thus support the idea that, in some situations, people are aware of at least some of the reasons propositions are considered true or false.

Finally, there is the question of whether associative learning effects depend on awareness of the influence that the process for generating and evaluating propositions has on behavior. When people are simply asked to judge how or how closely events are related (as is the case in many studies on causal and contingency learning in humans), it seems obvious that they are aware that their judgment is the result of their propositional beliefs about the relation between the events. They might not know how the relevant proposition was generated or evaluated (see previous paragraph), but they are probably aware that their causal or contingency rating is an expression of their conscious propositional belief about the relation between the events. It is less clear whether other associative learning effects also occur only if participants are aware that a particular proposition drives their behavior; take the example of human electrodermal conditioning. Does a conditioned change in skin conductance occur only if participants are aware that the change is the result of their propositional knowledge about the relation between the CS and US? It seems unlikely that participants are aware that propositional knowledge can change skin conductance levels. They are perhaps even unaware of the changes in skin conductance. Nevertheless, conditioned changes in skin conductance are compatible with a propositional view on learning, if one accepts the idea that propositional knowledge can have automatic effects on cognition, emotion, and behavior (see Lovibond \& Shanks, 2002, p. 4). This idea seems quite plausible. Imagine, for instance, that you are convinced that a tiger is about to enter the room that you are in. Once you have evaluated this proposition as being true, it will be difficult to stop thinking about the tiger, to control your fear, and to control attempts to escape.

It should be clear that more research is needed on whether and how associative learning is related to awareness of the process responsible for the generation and evaluation of propositions and awareness of the effects of that process on behavior. Along with Shanks (2007), I believe that a lot can be learned about associative learning by asking participants to justify or explain the observed associative learning effects (also see De Houwer, Baeyens, \& Field, 2005, p. 170). Although there are methodological issues to consider (e.g., Ericsson \& Simon, 1993; Smith \& Miller, 1978), justification can inform us about whether participants were aware of crucial events, their relation, and the effect of this relation on their behavior. Associative learning effects that can be correctly justified by the participant may well be driven by different procedural factors than associative learning effects that cannot be justified; nevertheless, the presence or absence of a correct justification cannot be used as a criterion for determining whether the associative learning effect is driven by propositions. An incorrect justification can result either from the lack of awareness of (1) the relation between the events (i.e., the output of the process) and thus the events themselves (i.e., the input of the process); (2) the factors that led to the formation and truth evaluation of the relevant proposition; or (3) the proposition that was responsible for the associatively induced change in behavior. Although this will be discussed in more detail later on in this article, propositional models do not necessarily imply that association learning depends on awareness in the sense of awareness of the process or its effect. Hence, such models do not lead to the prediction that people can always justify or explain associative learning effects; they do postulate, however, that associative learning depends on awareness of the input and output of the process, an assumption that seems to be supported by the available evidence.

Cognitive resources. A second feature of automaticity refers to the extent to which the operation of a process depends on the presence of cognitive resources. The word efficient is often used for a process that requires few resources, whereas the word effortful is used to describe a process that can operate only if many resources are available. Hence, efficiency is not a categorical, all-or-none property, but a dimensional feature, ranging from very efficient to very effortful (Moors \& De Houwer, 2006). Because cognitive resources are assumed to be limited, the operation of one effortful process will interfere with the operation of another effortful process. Therefore, if associative learning depends on an effortful process (as is postulated by propositional models), learning effects should be influenced by the mental load imposed by a secondary task present during the learning task. Research by Dawson and colleagues indeed confirmed that the presence of a demanding secondary task reduces the magnitude of associative learning in humans (e.g., Dawson, 1970; Dawson \& Biferno, 1973).

One could argue that the findings of Dawson and colleagues (Dawson, 1970; Dawson \& Biferno, 1973) fail to support any particular theoretical view, because all possible processes (including association formation processes) might be to some extent effortful. A recent finding by De Houwer and Beckers (2003), however, does provide more selective evidence for propositional models. When exposed to $\mathrm{A}+$ and $\mathrm{AX}+$ trials, it is reasonable to assume that the evaluation of the $\mathrm{X}$-outcome relation requires more complex and thus effortful processes than the evaluation of the $\mathrm{A}$-outcome relation. The latter requires only knowledge of the $\mathrm{A}+$ trials and the fact that the outcome does not occur on its own, whereas the former requires a comparison of the outcome on $\mathrm{A}+$ and $\mathrm{AX}+$ trials, while taking into account possible ceiling effects. Hence, mental load should have a bigger effect on the causal ratings for $\mathrm{X}$ than on the causal ratings for A. In fact, mental load should increase the ratings for $\mathrm{X}$, because it should interfere with the inference that $X$ is not a cause of the outcome: This was indeed observed. The fact that an increase in secondary task load can increase conditioned responding to one 
cue but leave conditioned responding to another cue unaffected or lessened (see Dawson, 1970) is very difficult to explain on the basis of association formation models (see Vandorpe \& De Houwer, 2006, for more data).

Time. The need for time is a dimensional feature, in that all processes require a certain amount of time and differ only in the exact amount of time that they need in order to run to completion. Some processes are relatively fast, some are relatively slow. Nevertheless, the relative speed of a process is a central feature of automaticity (Moors $\&$ De Houwer, 2006). For instance, the time needed to complete a process is partly determined by the extent to which it requires cognitive resources. In its turn, the speed of a process determines whether it is possible to control it or to become aware of it. Propositional models postulate that the generation and evaluation of propositions is controlled, in the sense that it requires time in order to complete (e.g., De Houwer et al., 2005, p. 245). This does not mean that the generation and evaluation of propositions will always be slow; if necessary, it can be done expeditiously. Also, once a proposition has been generated or evaluated, the evaluation can be stored in memory and quickly retrieved in later situations similar or identical to the present situation. One can predict, however, that imposing a time constraint on the generation and truth evaluation of a new proposition will decrease the number of factors (e.g., propositions about possible ceiling effects) taken into account when the proposition is evaluated. Another prediction is that, when no time constraints are imposed, the time that participants take to evaluate a new proposition will depend on the number of factors taken into account. Surprisingly little research has been conducted to test predictions like these. One exception is a study by Vandorpe, De Houwer, and Beckers (2004), who observed that participants took almost twice as long after $\mathrm{A}+\mathrm{AX}+$ and $\mathrm{B}-\mathrm{BY}+$ trials to give contingency ratings for cues $\mathrm{X}$ and $\mathrm{Y}$ as they did for cues $\mathrm{A}$ and $\mathrm{B}$. This finding is in line with the second prediction that I discussed. More research on this topic is therefore needed.

Goals. Automatic processes are often said to be unintentional, uncontrolled, autonomous, goal independent, or purely stimulus driven. Moors and De Houwer (2006) pointed out that all of these features are related to goals. The features unintentional, uncontrolled, and autonomous refer to the goals of the process being described (so-called proximal goals); these are the goals to start, stop, alter, or avoid the operation of the process. A process can be called unintentional if it can operate even in the absence of the goal to start the process. A process can be uncontrolled in that it is unaffected by goals to start, stop, alter, or avoid the process. If a process is uncontrolled with regard to all proximal goals, it can be called autonomous. Goalindependent processes are processes independent not only of proximal goals, but also of other goals unrelated to the process itself. Purely stimulus-driven processes, finally, are not only goal-independent but also independent of other factors such as awareness or attention (see Moors \& De Houwer, 2006, for more details).

Proponents of propositional models often refer to the generation and evaluation of propositions about relations as being a controlled process (e.g., De Houwer et al., 2005). This would imply that propositions are generated and evaluated only if organisms have the goal of doing so and do not have the goals of stopping or avoiding the process. Also, they should be able to alter the outcome of the generation and evaluation of propositions at will. Although these are central assumptions in a propositional approach, there is little or no research aimed specifically at testing these ideas. Assuming that the goal of learning necessarily involves the goals of generating and evaluating propositions about relations in the world, studies on incidental learning (i.e., learning without the goal to learn) could be regarded as relevant. Although there certainly is evidence for incidental learning, in many studies on incidental learning, learning effects are measured by asking participants whether certain events occurred. As such, the goal of learning is still induced, but in this case during the test phase, after the presentation of the events to be learned about. Because propositions about relations can be generated and evaluated equally well on the basis of memories of past events as on the basis of currently experienced events, most studies on incidental learning tell us little about whether propositions are generated and evaluated only if the goal to do so is present. There are, of course, many studies on associative learning in which (1) learning effects are measured indirectly (i.e., without asking participants to evaluate events but using nonverbal responses such as reaction times or physiological responses) and (2) participants have not been instructed to learn associations. Nevertheless, even in these studies, participants could have spontaneously formed the goal of learning about relations between events, for instance, because it was important for them to predict certain (biologically significant) events. ${ }^{5}$ Because this is rarely checked, to the best of my knowledge there still is no conclusive evidence for associative learning in the absence of the goal of learning.

Even if the pitfalls described in the previous paragraphinducing goals through test instructions, and the spontaneous generation of goals - are avoided, it will remain difficult to demonstrate learning in the absence of the goal of learning, simply because it is always difficult to demonstrate that a certain goal (or anything else for that matter) is completely absent. A more realistic approach might be to examine whether the likelihood or magnitude of associative learning effects depends on the extent to which organisms have the goal of learning. There is clear evidence for such a relation. For instance, Baeyens, Eelen, and Van den Bergh (1990) found that participants consciously detected about four times as many relations between events when they were instructed to detect such relations than when they were not instructed to. This clearly accords with the hypothesis that associative learning depends on the goal of learning about relations in the world, as predicted by propositional models. Interestingly, Baeyens et al. also observed that associatively induced changes in the liking of the stimuli were unaffected by instructions to learn relations between events (see also Hammerl \& Fulcher, 2005). This suggests that not all associative learning effects might depend to the same extent or in the same way 
on the goal of learning. I will return to this issue in the third and final part of the article.

\section{PART III \\ What Are the Unique Merits of a Propositional and Association Formation Approach to Associative Learning?}

In the previous section, I described the predictions that can be derived from the propositional approach to associative learning and summarized the evidence regarding these predictions. They were related to the factors that influence associative learning effects (prior knowledge, beliefs about experienced events, instructions, intervention, deductive reasoning) and the conditions under which these factors influence associative learning effects (awareness of events and relations, availability of cognitive resources, availability of time, and presence of proximal goals). None of the predictions originated from traditional association formation models. Hence, a first major conclusion of this article is that the propositional approach to associative learning has contributed a lot to the understanding of associative learning and still has a lot more to offer in terms of predictions still remaining to be examined in detail. This does not imply, however, that findings in support of the predictions of propositional models cannot also be explained by certain association formation models. It needs to be considered whether the propositional approach to associative learning makes a unique contribution to the understanding of associative learning completely beyond the scope of association formation models. The fact that the propositional approach has contributed a lot to the study of associative learning does not also imply that association formation models cannot make a unique contribution, beyond the scope of propositional models, to the study of associative learning. In the remainder of this article, I will discuss these two remaining questions: (1) What is the unique contribution of propositional models to our understanding of associative learning? (2) What is the unique contribution of association formation models to our understanding of associative learning?

\section{What Is the Unique Contribution of Propositional Models to Our Understanding of Associative Learning?}

Although many of the findings discussed in this article cannot be explained by existing association formation models, it would be tricky to claim that certain findings are entirely beyond the scope of this class of models. At this moment in time, there are already many different association formation models that each incorporate a variety of parameters. Different models or versions of models often predict opposite effects, making it very difficult to disprove the entire class of association formation models (see Miller \& Escobar, 2001). The problem is aggravated by the fact that researchers do not hesitate to adjust models in a post hoc manner (e.g., Dickinson \& Burke, 1996; Melchers et al., 2004; Van Hamme \& Wasserman, 1994). Also, it is difficult to foresee future developments in association formation models. Although at least some researchers acknowledge that association formation models probably cannot account for all aspects of associative learning (e.g., Dickinson, 2001; Shanks, 2007), it would be ill advised to claim that a particular finding is completely beyond the scope of association formation models and provides unique support for propositional models.

The evidence reviewed in this article does, however, put into focus the core challenges that association formation models face. First, they ignore the structure of relations in the world (Lagnado et al., 2007). Associations specify the extent to which events are related but not the way in which they are related. Hence, association formation models fail to take into account a host of factors that provide information about the structure of relations and that clearly influence associative learning effects (e.g., prior knowledge, instructions, intervention, deductive reasoning). Second, it is clear that associative learning at least in part depends on nonautomatic processes. If association formation models want to capture this, they need to drop the long-lived assumption that association formation is, by and large, an automatic process (e.g., Le Pelley, Oakeshott, \& McLaren, 2005, p. 65; McLaren, Green, \& Mackintosh, 1994, p. 321).

Parallel distributed processing (PDP) models might be able to meet these challenges. PDP models, however, differ in a fundamental manner from the association formation models discussed in this article. In association formation models, information about events and their relation is stored in a symbolic manner. A node represents a stimulus or event, and the link between the nodes represents the strength of the relation between stimuli or events. In PDP models, on the other hand, knowledge is represented in a distributed manner. An individual node or link does not represent anything as such. PDP models can be seen as artificially constructed miniature brains in which the nodes are simulated neurons and the links are simulated dendrites (Clark, 1990). As is the case with the brain, knowledge about how information is represented and transformed within a PDP network can be inferred only from observing how it responds to stimuli and simulated lesions. In terms of Marr's (1982) levels of explanation, PDP models thus provide an explanation at a simulated implementational level, whereas association formation and propositional models provide explanations at the algorithmic level. In principle, both association formation models and propositional models can be implemented in PDP networks. If a PDP model can be constructed that responds as if it has propositional knowledge that is formed in a nonautomatic manner, this would not show that the findings discussed in this article can be explained by (symbolic) association formation models.

Perhaps association formation models will at some point in time meet the challenges that were put forward in this article. Even if this does happen, propositional models have the merit that they dramatically increased our functional understanding of associative learning. Again referring to Marr's (1982) levels of explanation, functional knowledge is concerned with understanding what elements determine behavior, whereas algorithmic understanding refers to how those elements determine behavior. ${ }^{6}$ Both propositional 
and association formation models go beyond the functional level, in that they incorporate assumptions about the psychological processes by which a certain input is translated into a certain behavior. However, from a functional point of view (e.g., Baum, 1994; James, 1907/1974), one could argue that the only merit of algorithmic models is to organize and increase knowledge at the functional level. A functionalist wants to know what determines behavior, not how it is determined. For a functionalist, it therefore does not matter whether association formation models might at one time be capable of explaining how elements like prior knowledge or instructions influence learning effects; what is important is that propositional models are at the moment the most interesting ones, because they lead to a wealth of new functional knowledge. Hence, propositional models should be explored. The success of future association formation models will be determined not by whether they can explain the same functional knowledge as propositional models do, but by whether they generate new valid functional knowledge. In the next section, I will consider the unique insights that association formation models might offer.

\section{What Is the Unique Contribution of Association Formation Models to Our Understanding of Associative Learning?}

Association formation models can account for associative learning effects that are beyond the scope of propositional models. As mentioned earlier, even some proponents of association formation models doubt whether the latter would be able to account for the same results as propositional models would. Shanks (2007, pp. 297-298), for instance, recently argued that

It is important to realize that when arguing for a contribution of associative processes, supporters of this approach have never denied that rational causal thinking takes place. Of course, this would be absurd. . . Rather, the question is whether all causal thought is of this form, or whether instead there might be a separate type of thinking (associative) when people make intuitive judgments under conditions of less reflection.

Likewise, McLaren et al. (1994) "agree there exist two qualitatively different types of learning" (p. 315) that are based on "an associative system which cumulates information about contingencies between events and a cognitive system with beliefs and reasons for those beliefs" (p. 327). These authors are thus arguing for a dual-process model of associative learning, in which both propositional and association formation processes play a role. A dual-process model, however, makes sense only if one can specify what aspects of associative learning can be explained strictly on the basis of association formation processes. In the following paragraphs, I will consider a number of possibilities.

As can be inferred from the quote printed above, Shanks (2007) argued that association formation processes drive changes in behavior emitted intuitively - that is, "under conditions of less reflection." He specified that "if a par- ticipant agrees, on reflection, that a judgment was nonnormative or irrational, we can regard it as driven by intuition" (p. 298). The problem with this criterion is that it can also be fulfilled if behavior is driven by the truth evaluation of propositions. A person might initially fail to take into account a certain argument when evaluating the truth of a proposition and act according to the incorrect outcome of the truth evaluation process. That person might afterward realize that the initial truth evaluation was incorrect, but this would not change the fact that behavior was based on the truth evaluation of a proposition. This argument also clarifies that propositional models of associative learning are not incompatible with the existence of learning effects that seem to defy a rational explanation (see Karazinov \& Boakes, 2007, and Le Pelley et al., 2005, for examples). The fact that learning is based on reasoning does not mean that people always reason in a normatively correct manner and always take into account all relevant information. This insight does not render propositional models unfalsifiable. If the irrational learning effect is due to normatively incorrect reasoning, the effect should change when participants are encouraged to reason in a normatively correct manner (see Le Pelley et al., 2005, for a result that seems to defy this prediction). In sum, it is not the case that only association formation models can account for learning effects that seem irrational.

What about learning effects that a participant cannot justify at all, be it in a rational or irrational manner? ${ }^{7}$ Do these effects fall beyond the scope of propositional models? There are reasons to suggest that they do not. Behavior might be based on the truth evaluation of a proposition, even if people cannot explain why they reached a certain conclusion regarding the truth of a proposition. It seems reasonable to assume that people often generate and evaluate the truth of propositions on the basis of hunches that they cannot justify. Even in those cases, learning crucially depends on the generation and evaluation of propositions. A propositional view on learning and behavior is compatible with the idea that different kinds of arguments are taken into account when propositions are being generated and evaluated: Arguments that can be made explicit and those that cannot be (Gawronski \& Bodenhausen, 2006). In fact, modern theories of reasoning do make the distinction between an implicit (also called System 1) and an explicit (also called System 2) type of reasoning (e.g., J. Evans, 2003). Both types of reasoning might be based on different processes. However, a dual-process model of reasoning does not imply a dual-process model of associative learning. One can maintain the hypothesis that learning always depends on the generation and evaluation of propositions, even if one accepts the idea that the generation and evaluation of propositions might be based on either implicit or explicit forms of reasoning.

The idea that the generation and evaluation of propositions can be based on different types of reasoning does lead to the question of how these types of reasoning differ. Assume that implicit reasoning, in part, depends on association formation processes. In that case, association formation would play a role in associative learning, albeit by influencing the generation and evaluation of propo- 
sitions. Although it is difficult to refute the possibility that association formation processes influence learning in this manner, it is important to point out that such is not necessarily the case; a reasonable alternative is that implicit reasoning depends on the automatic activation of memory traces. Research on implicit memory has demonstrated that memories can be activated automatically, in the sense of unconsciously, efficiently, quickly, and independently of proximal goals (e.g., Richardson-Klavehn, Lee, Joubran, \& Bjork, 1994). The automatic storage and retrieval of memory traces does not necessarily rely on the formation of abstract associations between representations in memory. An alternative view is that each experienced event is stored in a separate memory trace (called instances or episodes) that can subsequently be activated automatically as a function of the similarity between the past and present situation (e.g., Hintzman, 1986; Medin \& Schaffer, 1978; Nosofsky \& Palmeri, 1997). In this way, the processes involved in perception and memory are sufficient to account for unjustifiable intuitions about the truth of propositions.

If these intuitions are taken into account during the generation and evaluation of propositions, irrational behavior could emerge. In addition to errors in explicit reasoning, implicit reasoning is thus a second source of irrational behavior in propositional models of learning. A crucial test for propositional models, therefore, is not whether learning experiences can have irrational effects on behavior, but whether a relation in the world can influence behavior even if the organism did not at some point in time represent the relation in a propositional form. ${ }^{8}$ The qualification "at some point in time" was added because once a relation has been represented in a propositional format, it can be stored as such in memory and can be activated automatically. In such cases, having experienced a relation can influence behavior, even when a proposition about the relation is not entertained. This could explain how CSs can produce a conditioned response even if they are presented subliminally when the CS-US relation was previously detected consciously (Flykt, Esteves, \& Öhman, 2007). The automatic activation of memory traces containing previously entertained propositions could also lead to feelings of ambivalence when activated old beliefs contradict current beliefs. Finally, note that an instance-based view of memory is compatible with the fact that new learning does not imply unlearning of old information (Bouton, 1993).

The idea that irrational and unjustifiable changes in behavior can be due only to association formation processes is built on the incorrect argument that the generation and evaluation of propositions can produce only rational and justifiable behaviors. A different criterion for excluding an impact of propositional processes is whether the behavior itself can be influenced by propositional processes. If a particular behavior cannot be affected by propositional beliefs, it follows that associatively induced changes in this behavior cannot be due to the generation and evaluation of propositions. Although this argument is valid, it can be used only if there are sound arguments for the claim that a particular behavior cannot be influenced by propositional processes. This needs to be examined carefully. For instance, it should be clear that not only verbal but also nonverbal responses can be modulated by propositional processes. It is well known that physiological responses, such as skin conductance, rely heavily on propositional knowledge (e.g., Lovibond, 2003). Also, neurobiological responses, such as dopamine release, are probably susceptible to propositional beliefs (e.g., conscious beliefs about causes of reward). One also cannot simply assume that so-called implicit measures of associations in memory are impervious to the impact of propositional beliefs (e.g., De Houwer, 2006). Finally, if one could show that a certain response cannot be influenced by propositional processes, and if one could change this response as the result of relations in the environment, this would contradict propositional models but would not necessarily support association formation models. Evidence against one account does not necessarily provide evidence for another account. It might well be that processes other than association formation underlie those associative learning effects (e.g., the automatic activation of episodic memory traces).

Whereas the nonverbal nature of a response does not necessarily exclude a contribution of propositional processes, one could argue that associative learning effects in animals cannot be due to propositional processes, simply because animals are incapable of generating and evaluating propositions about relations in the world. This argument is not problematic for propositional models, if these models were applied only to humans, but it might be premature to exclude animal learning from the scope of propositional models. There are indications that at least some animals are capable of representing knowledge about relations in the world in a propositional manner. For instance, using a design similar to that of Waldmann and Hagmayer (2005), Blaisdell, Sawa, Leising, and Waldmann (2006) showed that rats are sensitive to the distinction between intervention and observation. This strongly supports the hypothesis that rats represent the causal nature of relations in the world (see also Testa, 1974). Likewise, the data of Beckers, Miller, De Houwer, and Urushihara (2006) suggest that blocking effects in rats are moderated by information about the additive effect of cues. Again, this makes sense from the perspective that rats take into account not only the strength of relations but also their structure (e.g., whether a relation is causal). Recent research has also shown that animals possess often astonishing cognitive capabilities (e.g., Clayton \& Dickinson, 1998). As Lovibond (2004) pointed out, given the evolutionary significance of associative learning, it seems likely that animals will use all the cognitive abilities that they have available in order to detect and respond to regularities in the environment. Although there might be qualitative differences in cognitive capacities between different types of animals, it seems reasonable to assume that at least some animals are capable of representing the world in such a way that they do not only code that events are related but also how events are related. If this assumption is reasonable, it is also reasonable to examine empirically the extent to which associative learning in animals might be based on the generation and evaluation of propositions. This ap- 
proach could lead to many important new insights, just as the propositional approach led to many new insights in human associative learning.

It has to be acknowledged that associative learning can be observed in species of animals that most likely do not engage in propositional processing. With neurologically unsophisticated species such as aplysia (a kind of snail), the entire neural circuitry involved in certain associative learning effects has been discovered, showing that changes in behavior are due to the formation and strengthening of dendrites between certain neurons (e.g., Krasne \& Glanzman, 1995). It is indeed unlikely that these learning effects are due to propositional processes. However, they do not necessarily inform us about the psychological processes involved in associative learning effects in humans and other species of animals. A first point to note is that, just like propositional models, modern formulations of association formation models are algorithmic theories of learning; that is, they are concerned with the psychological processes that translate certain patterns of input (experiences) into certain patterns of output (behavior). More specifically, association formation models postulate that associatively induced changes in behavior occur because associations are formed between representations in memory. Association formation models do not provide an explanation at the implementational level - that is, at the level of neurophysiological or neurobiological processes. Neurons are not representations. Dendrites are not associations through which activation can spread. To put it differently, just like propositional models, association formation models are software, not hardware. Hence, both types of models operate at the same level of explanation.

Although there is a certain analogy between psychological concepts such as representations, associations, and activation, and neurological concepts such as neurons, dendrites, and neurotransmitters, there is no one-to-one mapping between these concepts. Neurophysiological and neurobiological data can certainly provide inspiration for, and constrain, algorithmic theories about the psychological processes involved in associative learning effects, but algorithmic theories operate at a different level of explanation. Just as the functional and algorithmic levels of explanation need to be distinguished because any particular functional (input-output) relation can be explained on the basis of different algorithmic theories, the algorithmic and implementational levels need to be distinguished, because different algorithmic theories could provide an explanation for the function of a particular neurological structure or process. Therefore, observing the formation of dendrites between neurons as the result of the pairing of stimuli cannot be seen as the ultimate proof for the validity of association formation models. In fact, one can argue that associative learning effects not mediated by psychological processes and representations fall beyond the scope of both propositional models and association formation models. The formation of dendrites is such an effect; it can be explained at the implementational, neurological level, without referring to the algorithmic (processes) or functional (behavior) level.
There is also a second reason why information about associative learning in neurologically unsophisticated animals does not necessarily inform us about the processes underlying associative learning in humans and other neurologically more sophisticated animals. Different animals have evolved in different ways. Each animal needs to detect and respond to regularities in the environment, but different species probably use different processes to meet this challenge (Lovibond, 2004). If associative learning effects in humans depended on the same simple neurological changes as do associative learning effects in simple organisms, the question of why human brains have become so complex would arise. One could argue that an old evolutionary learning process, such as that observed in aplysia, has been supplemented by other learning processes during evolution but still operates in humans today. Such a hypothesis is, however, impossible to refute, absent criteria to specify when such a process would operate and influence human behavior. As was discussed earlier in this section, neither the irrational nor the nonverbal nature of the behavior seems to be a good criterion.

In sum, it is not clear whether there are associative learning effects that can be explained only on the basis of association formation models. Irrational learning effects can be the result of propositional processes. Learning in some nonhuman species of animals could rely on the generation and evaluation of propositions. Learning in neurologically unsophisticated organisms, or neurological effects of relations between events, might not be mediated by any psychological process, and thus falls beyond the scope of both propositional models and association formation models.

Only association formation models provide formalized (and thus precise) accounts of associative learning. Even if one agreed that association formation models do not explain more than can be explained on the basis of propositional models alone, one could still stick to association formation models, because they provide a more elegant explanation of associative learning effects. For many psychologists, "elegant" primarily means "using a simple mathematical formula." Elegance could be operationalized as the ratio between the number of findings that the model can explain and correctly predict, on one hand, and the number of parameters needed to be postulated in order to explain and predict those findings, on the other. The advantage of mathematical models is that it can be clarified in detail how the model accounts for known results and why it leads to certain predictions. Many association formation models have indeed been formulated in mathematical terms. Some, like the wellknown Rescorla-Wagner (1972) model, are very elegant in that, on the basis of a very simple model containing only a few parameters, they can explain many findings. Nevertheless, two arguments can be made against the idea that association formation models should be preferred to propositional models because they can be formalized mathematically. (1) The precision of formalized association formation models is to a large extent an illusion; and 
(2) propositional as well as association formation models can be formalized. I will elaborate on these arguments in the next paragraphs.

With regard to the first point, it is correct that mathematically formalized models can provide an explicit account for particular findings. The problem is, however, that each explanation depends on a host of hidden assumptions regarding the meaning and value of the parameters in the model and the conditions under which the value of parameters changes. Often, one and the same model can explain opposite results, depending on the value of a particular set of parameters. This becomes even more problematic if parameters are added to the model in order to accommodate results that could not be explained by the original model. In such cases, it is often not clear whether the revised model can explain all the findings that the original model could. Because of this flexibility, association formation models lose their precision and become difficult to refute. This is especially true when different variants of different association formation models are used to explain different findings. The class of association formation models as a whole allows for no precision at all, because almost every possible result can be explained by some version of some model. Even if one takes seriously one particular explanation of a particular finding, it is not always clear what the explanation actually means. In order to have more than a mathematical explanation of behavior, it is necessary to relate the parameters in a mathematical model to psychological constructs (e.g., knowledge, processes). When this is not made explicit, the model loses precision. Even if the psychological meaning of parameter values is made clear, sometimes one is forced to accept parameter values that seem incompatible with the proposed psychological meaning of the parameter (e.g., negative salience; see Van Hamme \& Wasserman, 1994). In sum, formalized association formation models are often not as precise as they seem to be.

Second, the propositional approach to associative learning can, in principle, also be formalized. First of all, it is important to realize that existing mathematical models of associative learning are not necessarily incompatible with a propositional approach (Lovibond, 2003, 2004). Mathematical formulas as such specify only which inputs will lead to which outputs. They do not incorporate assumptions about how knowledge is represented and processed. Hence, in terms of Marr's (1982) levels of explanation, mathematical models operate at the functional rather than at the algorithmic level. It is only when mathematical models are assumed to describe the formation of associations in memory that they become algorithmic. If this assumption is dropped, mathematical models of learning become perfectly compatible with a propositional approach, because both operate at a different level of explanation. One could even argue that mathematical formulas, such as the Rescorla-Wagner (1972) learning rule, capture the operating principles of propositional processes (Lovibond, 2003). Hence, researchers who do not want to give up the (apparent) precision of mathematical models can continue to use these models, even if they abandon the idea that associative learning is based on the formation of associations in memory.

Propositional models are compatible not only with many of the traditional mathematical models of associative learning but also with the more recent Bayesian approach of human cognition (see Chater, Tenenbaum, \& Yuille, 2006, for an introduction). In fact, Bayesian models provide a formalization of some of the core ideas in propositional models. First of all, Bayes nets offer a way to represent propositions in a formal manner. Bayes nets can be seen as mental models of the world that specify how events are causally related. Hence, they represent not only the fact that events are related (i.e., strength) but also how they are related (i.e., structure) - more specifically, which events are causes of which other events. The structure of a Bayes net is determined not only by experience. In fact, it is explicitly acknowledged that experiencing the presence and absence of events is, as such, a poor indicator of the structure of relations (e.g., Pearl, 2000). Rather, beliefs about the structure of Bayes nets are assumed to be determined primarily by other factors, such as instructions, prior knowledge, and intervention (see Lagnado et al., 2007). Second, the inferences that underlie beliefs about the structure of relations in the world can be formalized using Bayesian inference rules. Hence, the combination of Bayes nets and Bayesian inference offers a way to formalize the manner in which certain propositions are represented, generated, and evaluated.

Although the Bayesian approach already has many strengths (e.g., the representation of degrees of belief), at present it does not capture all aspects of a propositional approach. Most importantly, it does not capture the idea that propositional processes are nonautomatic. In fact, it does not offer any realistic perspective on the processes that humans actually use to generate or evaluate propositions. Bayesian inferences are computationally so complex and demanding that it is impossible that humans actually use them. Although researchers are working to resolve these problems (e.g., Tenenbaum, Kemp, \& Shafto, 2007), it remains useful to continue to entertain less formalized propositional models, such as causal model theory (e.g., Waldmann, 2000).

\section{SUMMARY AND CONCLUSIONS}

In the present article, I have tried to explain why it is important to consider models of associative learning that do not rely on the idea of association formation, what propositional models entail and why they provide a good alternative for association formation models, and whether there are still reasons to retain the idea that association formation is a process underlying associative learning effects. I argued that associative learning should be regarded as an effect; that is, a change in behavior attributed to relations in the world. There is no a priori reason to assume that associative learning effects can be produced only by the formation of associations in memory. One should be free to consider any possible theoretical explanation of associative learning effects. A possible alternative expla- 
nation for associative learning effects is offered by propositional models. The core assumptions of these models are that (1) associative learning effects are based on the generation and evaluation of propositions about relations in the world, and (2) nonautomatic processes intervene in the generation and evaluation of these propositions. In line with the first assumption, studies have shown that factors that influence the generation and evaluation of propositions also influence associative learning effects. In support of the second assumption, it has been demonstrated that associative learning effects depend on nonautomatic processes. Many of the findings in support of propositional models cannot be explained on the basis of existing association formation models. Although future association formation models may be able to explain these findings, the evidence summarized in this article clearly provides a challenge for this class of models. Whereas propositional models thus seem to offer a unique contribution to our understanding of associative learning, it is less clear whether particular findings can be explained only on the basis of association formation models. Contrary to claims made in the literature (e.g., Shanks, 2007), irrational learning effects and associative learning in (certain) animals does not necessarily fall beyond the scope of propositional learning models. Also, the formalized nature of many association formation models is not a sufficient reason to cling to the idea of association formation.

Readers, having come to the end of this article, will probably notice that I have focused on the strengths of the propositional approach and the potential weaknesses of the association formation approach. Although I also tried to respond to potential criticisms of the propositional approach, I realize that it will be difficult for associative learning researchers to give up the association formation models that have served them so well over the past 100 years. I do hope, however, that the reader will agree with the following two general conclusions.

First, despite the current shortcomings of propositional models, research that was inspired by these models has led to many important new insights in associative learning. Associative learning effects are driven not only by the direct experience of events; prior knowledge, instructions, intervention, and deductive reasoning also play a part. The processes that underlie associative learning effects are in many respects nonautomatic. The fact that these insights do not fit well with the association formation approach does not mean that they are not important. The aim of learning psychologists should be to understand associative learning effects rather than to understand association formation or just those aspects of associative learning effects that fit with the association formation approach. Hence, I hope the reader will agree that the propositional approach to associative learning is useful.

Second, although it might be too early to entirely give up the idea of association formation, I hope that researchers will give up the idea that associative learning effects are by default due to association formation processes. Not only is there no logical necessity that associative learning effects must be due to the formation of associations between representations in memory; such an assumption hampers theoretical progress regarding our understanding of associative learning effects and is difficult to reconcile with a substantial part of the relevant empirical data. Rather than assuming the operation of association formation processes by default, researchers should try to carefully justify why association formation would underlie a particular associative learning effect. Detailed criteria are needed that can be used to determine when association formation processes drive associative learning effects. The validity of these criteria of course needs to be supported by sound arguments and empirical data. Without such criteria, the association formation approach has little value, because there is no way to specify what the added value of this approach is. As such, I hope that the present article will provide an impetus for the development not only of propositional models but also of association formation models. Such developments will serve the ultimate goal of understanding associative learning effects.

\section{AUTHOR NOTE}

This article was made possible by Grants BOF-01100805 and BOF/ GOA2006/001 from Ghent University. I thank Geoff Hall for inviting me to write this article and the many researchers with whom I discussed the possible merits of propositional and association formation models, in particular Lorraine Allan, Tom Beckers, Tony Dickinson, Mike LePelly, Peter Lovibond, Helena Matute, Chris Mitchell, Ralph Miller, Agnes Moors, David Shanks, Stefaan Vandorpe, Tom Verguts, Michael Waldmann, and Andy Wills. Correspondence concerning this article should be addressed to J. De Houwer, Department of Psychology, Ghent University, Henri Dunantlaan 2, B-9000 Ghent, Belgium (e-mail: jan .dehouwer@ugent.be).

Note-This article is one of an occasional series of reviews invited by the editors.

\section{REFERENCES}

Aitken, M. R. F., Larkin, M. J. W., \& Dickinson, A. (2001). Reexamination of the role of within-compound associations in the retrospective revaluation of causal judgements. Quarterly Journal of Experimental Psychology, 54B, 27-51.

BACON, F. (1994). Novum organum. Chicago: Open Court. (Original work published 1620)

Baeyens, F., Eelen, P., \& Van den Bergh, O. (1990). Contingency awareness in evaluative conditioning: A case for unaware affectiveevaluative learning. Cognition \& Emotion, 4, 3-18.

BARGH, J. A. (1992). The ecology of automaticity: Toward establishing the conditions needed to produce automatic processing effects. American Journal of Psychology, 105, 181-199.

BAUm, W. M. (1994). Understanding behaviorism. New York: HarperCollins.

Beckers, T., De Houwer, J., Pineño, O., \& Miller, R. R. (2005). Outcome additivity and outcome maximality influence cue competition in human causal learning. Journal of Experimental Psychology: Learning, Memory, \& Cognition, 31, 238-249.

Beckers, T., Miller, R. R., De Houwer, J., \& Urushihara, K. (2006). Reasoning rats: Forward blocking in Pavlovian animal conditioning is sensitive to constraints of causal inference. Journal of Experimental Psychology: General, 135, 92-102.

Blaisdell, A., Sawa, K., Leising, K. J., \& Waldmann, M. R. (2006). Causal reasoning in rats. Science, 311, 1020-1022.

Bolles, R. C. (1979). Learning theory (2nd ed.). Oxford: Holt, Rinehart \& Winston.

Bouton, M. E. (1993). Context, time, and memory retrieval in the interference paradigms of Pavlovian learning. Psychological Bulletin, 114, 80-99.

Bouton, M. E. (2007). Learning and behavior. Sunderland, MA: Sinauer. 
Bullock, M., Gelman, R., \& Baillargeon, R. (1982). The development of causal reasoning. In W. J. Friedman (Ed.), The developmental psychology of time. New York: Academic Press.

Carson, S. H., Peterson, S. B., \& Higgins, D. M. (2003). Decreased latent inhibition with increased creative achievement in highfunctioning individuals. Journal of Personality \& Social Psychology, 85, 499-506.

Chater, N., Tenenbaum, J. B., \& Yuille, A. (2006). Probabilistic models of cognition: Conceptual foundations. Trends in Cognitive Sciences, 10, 287-291.

Cheng, P. W. (1997). From covariation to causation: A causal power theory. Psychological Review, 104, 367-405.

Clark, A. (1990). Connectionism, competence, and explanation. British Journal of Philosophical Science, 41, 195-222.

Clayton, N. S., \& Dickinson, A. (1998). Episodic-like memory during cache recovery by scrub jays. Nature, 395, 272-274.

Colgan, D. M. (1970). Effect of instructions on the skin conductance response. Journal of Experimental Psychology, 86, 108-112.

COOK, S. W., \& HARris, R. E. (1937). The verbal conditioning of the galvanic skin reflex. Journal of Experimental Psychology, 21 , 202-210

DAwson, M. E. (1970). Cognition and conditioning: Effects of masking the CS-UCS contingency on human GSR classical conditioning. Journal of Experimental Psychology, 85, 389-396.

Dawson, M. E., \& Biferno, M. A. (1973). Concurrent measurement of awareness and electrodermal classical conditioning. Journal of Experimental Psychology, 101, 55-62.

De Houwer, J. (2002). Forward blocking depends on retrospective inferences about the presence of the blocked cue during the elemental phase. Memory \& Cognition, 30, 24-33.

De Houwer, J. (2006). Using the implicit association test does not rule out an impact of conscious propositional knowledge on evaluative conditioning. Learning \& Motivation, 37, 176-187.

De Houwer, J. (2007). A conceptual and theoretical analysis of evaluative conditioning. Spanish Journal of Psychology, 10, 230-241.

De Houwer, J., Baeyens, F., \& Field, A. P. (2005). Associative learning of likes and dislikes: Some current controversies and possible ways forward. Cognition \& Emotion, 19, 161-174.

De Houwer, J., \& Beckers, T. (2002a). Higher-order retrospective revaluation in human causal learning. Quarterly Journal of Experimental Psychology, 55B, 137-151.

De Houwer, J., \& Beckers, T. (2002b). A review of recent developments in research and theory on human contingency learning. Quarterly Journal of Experimental Psychology, 55B, 289-310.

De Houwer, J., \& Beckers, T. (2002c). Second-order backward blocking and unrelease overshadowing in human causal learning. Experimental Psychology, 49, 27-33.

De Houwer, J., \& Beckers, T. (2003). Secondary task difficulty modulates forward blocking in human contingency learning. Quarterly Journal of Experimental Psychology, 56B, 345-357.

De Houwer, J., Beckers, T., \& Glautier, S. (2002). Outcome and cue properties modulate blocking. Quarterly Journal of Experimental Psychology, 55A, 965-985.

De Houwer, J., Beckers, T., \& VAndorpe, S. (2005). Evidence for the role of higher order reasoning processes in cue competition and other learning phenomena. Learning \& Behavior, 33, 239-249.

De Houwer, J., Thomas, S., \& Baeyens, F. (2001). Associative learning of likes and dislikes: A review of 25 years of research on human evaluative conditioning. Psychological Bulletin, 127, 853-869.

De Houwer, J., Vandorpe, S., \& Beckers, T. (2007). Statistical contingency has a different impact on preparation judgements than on causal judgements. Quarterly Journal of Experimental Psychology, 60, 418-432.

Denniston, J. C., Savastano, H. I., \& Miller, R. R. (2001). The extended comparator hypothesis: Learning by contiguity, responding by relative strength. In R. R. Mowrer \& S. B. Klein (Eds.), Handbook of contemporary learning theories (pp. 65-117). Mahwah, NJ: Erlbaum.

Dickinson, A. (1980). Contemporary animal learning theory. Cambridge: Cambridge University Press.

DicKInson, A. (2001). The 28th Bartlett memorial lecture: Causal learning: An associative analysis. Quarterly Journal of Experimental Psychology, 54B, 3-25.

Dickinson, A., \& BURKE, J. (1996). Within-compound associations me- diate the retrospective revaluation of causality judgements. Quarterly Journal of Experimental Psychology, 49B, 60-80.

Eelen, P. (1980). Klassieke conditioning: Klassiek en toch modern [Classical conditioning: Classic but nevertheless modern]. In J. R. Nuttin (Ed.), Gedrag, dynamische relatie en betekeniswereld [Behavior, dynamic relation, and world of meaning]. Leuven: Universitaire Pers Leuven.

Ericsson, K. A., \& Simon, H. A. (1993). Protocol analysis: Verbal reports as data (2nd ed.). Cambridge, MA: MIT Press.

Evans, J. (2003). In two minds: Dual-process accounts of processing. Trends in Cognitive Sciences, 7, 454-459.

Evans, M., Jamal, A., \& Foxall, G. R. (2006). Consumer behaviour. Chichester, U.K.: Wiley.

Flykt, A., Esteves, F., \& Öhman, A. (2007). Skin conductance responses to masked conditioned stimuli: Phylogenetic/ontogenetic factors versus direction of threat? Biological Psychology, 74, 328-336.

Garcia, J., \& Koelling, R. A. (1966). Relation of cue to consequence in avoidance learning. Psychonomic Science, 4, 123-124.

Gawronski, B., \& Bodenhausen, G. V. (2006). Associative and propositional processes in evaluation: An integrative review of implicit and explicit attitude change. Psychological Bulletin, 132, 692-731.

GaWronski, B., \& STRACK, F. (2004). On the propositional nature of cognitive consistency: Dissonance changes explicit but not implicit attitudes. Journal of Experimental Social Psychology, 40, 535-542.

Hammerl, M., \& Fulcher, E. P. (2005). Reactance in affectiveevaluative learning: Outside of conscious control? Cognition \& Emotion, 19, 197-216.

Hintzman, D. L. (1986). "Schema abstraction" in a multiple-trace memory model. Psychological Review, 93, 411-428.

Hume, D. (1987). A treatise of human nature (2nd ed.). Oxford: Oxford University Press, Clarendon Press. (Original work published 1739)

James, W. (1974). Pragmatism and four essays from The meaning of truth. New York: New American Library. (Originally published in 1907)

KAMIN, L. J. (1969). Predictability, surprise attention and conditioning. In B. A. Campbell \& R. M. Church (Eds.), Punishment and aversive behavior (pp. 279-296). New York: Appleton-Century-Crofts.

KANT, I. (1965). Critique of pure reason. London: Macmillan. (Original work published 1781)

Karazinov, D. M., \& Boakes, R. A. (2007). Second-order conditioning in human predictive judgments when there is little time to think. Quarterly Journal of Experimental Psychology, 60, 448-460.

Krasne, F. B., \& Glanzman, D. L. (1995). What we can learn from invertebrate learning. Annual Review of Psychology, 46, 585-624.

Lagnado, D. A., Waldmann, M. R., Hagmayer, Y., \& Sloman, S. A. (2007). Beyond covariation: Cues to causal structure. In A. Gopnik \& L. Schulz (Eds.), Causal learning: Psychology, philosophy, and computation (pp. 154-172). New York: Oxford University Press.

Le Pelley, M., Oakeshott, S., \& McLaren, I. (2005). Blocking and unblocking in human causal learning. Journal of Experimental Psychology: Animal Behavior Processes, 31, 56-70.

Lien, Y., \& CHENG, P. W. (2000). Distinguishing genuine from spurious causes: A coherence hypothesis. Cognitive Psychology, 40, 87-137.

Lopez, F. J., CoBos, P. L., \& CAÑo, A. (2005). Associative and causal reasoning accounts of causal induction: Symmetries and asymmetries in predictive and diagnostic inferences. Memory \& Cognition, 33, 1388-1398.

LoviBond, P. F. (2003). Causal beliefs and conditioned responses: Retrospective revaluation induced by experience and by instruction. Journal of Experimental Psychology: Learning, Memory, \& Cognition, 29, 97-106.

LoviBond, P. F. (2004, May). The role of additivity in blocking of causal judgment. Paper presented at the Special Interest Meeting on Human Contingency Learning, Le Lignely, Belgium.

Lovibond, P. F., Been, S.-L., Mitchell, C. J., Bouton, M. E., \& FroHARDT, R. (2003). Forward and backward blocking of causal judgment is enhanced by additivity of effect magnitude. Memory \& Cognition, 31, 133-142.

Lovibond, P. F., \& Shanks, D. R. (2002). The role of awareness in Pavlovian conditioning: Empirical evidence and theoretical implications. Journal of Experimental Psychology: Animal Behavior Processes, 28, 3-26.

LuCAS, E. J., \& BALL, L. J. (2005). Think-aloud protocols and the selec- 
tion task: Evidence for relevance effects and rationalisation processes. Thinking \& Reasoning, 11, 35-66.

Mackintosh, N. (1975). A theory of attention: Variations in the associability of stimuli with reinforcement. Psychological Review, 82, 276-298.

Maldonado, A., Catena, A., Perales, J. C., \& Cándido, A. (2007). Cognitive biases in human causal learning. Spanish Journal of Psychology, 10, 242-250.

MARR, D. (1982). Vision: A computational investigation into the human representation and processing of visual information. San Francisco: Freeman.

Matute, H., Arcediano, F., \& Miller, R. R. (1996). Test question modulates cue competition between causes and between effects. Journal of Experimental Psychology: Learning, Memory, \& Cognition, 22, 182-196.

McLaren, I. P. L., Green, R. E. A., \& Mackintosh, N. J. (1994). Animal learning and the implicit/explicit distinction. In N. C. Ellis (Ed.), Implicit and explicit learning of languages (pp. 313-332). London: Academic Press.

Medin, D. L., \& Schaffer, M. M. (1978). Context theory of classification learning. Psychological Review, 85, 207-238.

Meiser, T., Sattler, C., \& Weisser, K. (2008). Binding of multidimensional context information as a distinctive characteristic of remember judgments. Journal of Experimental Psychology: Learning, Memory, \& Cognition, 34, 32-49.

Melchers, K. G., Lachnit, H., \& Shanks, D. (2004). Within-compound associations in retrospective revaluation and in direct learning: A challenge for comparator theory. Quarterly Journal of Experimental Psychology, 57B, 25-54.

Miller, R. R., \& Escobar, M. (2001). Contrasting acquisition-focused and performance-focused models of acquired behavior. Current $\mathrm{Di}$ rections in Psychological Science, 10, 141-145.

Mitchell, C. J., KilledAR, A., \& LoviBOnd, P. (2005). Inference-based retrospective revaluation in human contingency judgments requires knowledge of within-compound relationships. Journal of Experimental Psychology: Animal Behavior Processes, 31, 418-424.

Mitchell, C. J., \& Lovibond, P. F. (2002). Backward and forward blocking in human electrodermal conditioning: Blocking requires an assumption of outcome additivity. Quarterly Journal of Experimental Psychology, 55B, 311-329.

Moors, A., \& De Houwer, J. (2006). Automaticity: A conceptual and theoretical analysis. Psychological Bulletin, 132, 297-326.

Nosofsky, R. M., \& PALmeri, T. J. (1997). An exemplar-based random walk model of speeded classification. Psychological Review, 104, 266-300.

Pearce, J. M., \& Hall, G. (1980). A model for Pavlovian learning: Variations in the effectiveness of conditioned but not of unconditioned stimuli. Psychological Review, 87, 532-552.

Pearl, J. (2000). Causality: Models, reasoning, and inference. Cambridge: Cambridge University Press.

Pleyers, G., Corneille, O., Luminet, O., \& Yzerbyt, V. (2007). Aware and (dis)liking: Item-based analyses reveal that valence acquisition via evaluative conditioning emerges only when there is contingency awareness. Journal of Experimental Psychology: Learning, Memory, \& Cognition, 33, 130-144.

Rescorla, R. A., \& Wagner, A. R. (1972). A theory of Pavlovian conditioning: Variations in the effectiveness of reinforcement and nonreinforcement. In A. H. Black \& W. F. Prokasy (Eds.), Classical conditioning II: Current research and theory (pp. 64-99). New York: Appleton-Century-Crofts.

Richardson-Klavehn, A., Lee, M. G., Joubran, R., \& Bjork, R. A. (1994). Intention and awareness in perceptual identification priming. Memory \& Cognition, 22, 293-312.

ShanKs, D. R. (2007). Associationism and cognition: Human contingency learning at 25. Quarterly Journal of Experimental Psychology, 60, 291-309.

Shanks, D. R., \& Darby, R. J. (1998). Feature- and rule-based generalization in human associative learning. Journal of Experimental Psychology: Animal Behavior Processes, 24, 405-415.

Smith, E. R., \& Miller, F. D. (1978). Limits on perception of cognitive processes: A reply to Nisbett and Wilson. Psychological Review, 85, 355-362.

Steyvers, M., Tenenbaum, J. B., Wagenmakers, E. J., \& Blum, B.
(2003). Inferring causal networks from observations and interventions. Cognitive Science, 27, 453-489.

Strack, F., \& Deutsch, R. (2004). Reflective and impulsive determinants of social behavior. Personality \& Social Psychology Review, 8 , 220-247.

Tenenbaum, J. B., Kemp, C., \& Shafto, P. (2007). Theory-based Bayesian models of inductive reasoning. In A. Feeney \& E. Heit (Eds.), Inductive reasoning. Cambridge: Cambridge University Press.

Testa, T. J. (1974). Causal relationships and the acquisition of avoidance responses. Psychological Review, 81, 491-505.

Vadillo, M. A., \& Matute, H. (2007). Predictions and causal estimations are not supported by the same associative structure. Quarterly Journal of Experimental Psychology, 60, 433-447.

Vadillo, M. A., Miller, R. R., \& Matute, H. (2005). Causal and predictive-value judgments, but not predictions, are based on cueoutcome contingency. Learning \& Behavior, 33, 172-183.

VANDORPe, S., \& De Houwer, J. (2006). A comparison of cue competition in a simple and a complex design. Acta Psychologica, 122, 234-246.

Vandorpe, S., De Houwer, J., \& Beckers, T. (2004, May). New evidence in support of an inferential account of cue competition in human contingency learning. Paper presented at the Special Interest Meeting on Human Contingency Learning, Le Lignely, Belgium.

VANDORPe, S., De Houwer, J., \& BeCKers, T. (2005). Further evidence for the role of inferential reasoning in forward blocking. Memory \& Cognition, 33, 1047-1056.

Vandorpe, S., De Houwer, J., \& Beckers, T. (2007). The role of memory for compounds in cue competition. Learning \& Motivation, 38, 195-207.

Van Hamme, L. J., \& Wasserman, E. A. (1994). Cue competition in causality judgments: The role of nonpresentation of compound stimulus elements. Learning \& Motivation, 25, 127-151.

WAGNER, A. R. (1985). SOP: A model of automatic memory processing in animal behavior. In R. R. Miller \& N. E. Spear (Eds.), Information processing in animals: Conditioned inhibition (pp. 223-266). Hillsdale, NJ: Erlbaum.

WALDMANN, M. R. (2000). Competition among causes but not effects in predictive and diagnostic learning. Journal of Experimental Psychology: Learning, Memory, \& Cognition, 26, 53-76.

WaLdmann, M. R., \& Hagmayer, Y. (2005). Seeing versus doing: Two modes of accessing causal knowledge. Journal of Experimental Psychology: Learning, Memory, \& Cognition, 31, 216-227.

Waldmann, M. R., \& HolyoaK, K. J. (1992). Predictive and diagnostic learning within causal models: Asymmetries in cue competition. Journal of Experimental Psychology: General, 121, 222-236.

\section{NOTES}

1. This definition of associative learning differs from the popular definition of learning as a psychological process that can but does not necessarily influence behavior. It is true that relations in the world can be learned in a latent manner, in the sense that they do not have an immediate impact on behavior. From a functional perspective, however, if a certain relation does not produce any change in behavior under any set of conditions, there is no reason to reject the null hypothesis that the relation has not been learned. Hence, from a functional perspective, behavioral change is the ultimate criterion to determine whether learning has taken place. (Note that I use a broad definition of behavior that includes physiological and neurological responses.)

2. Examples of such properties are the order and timing of the CS and US on a trial (e.g., forward vs. backward conditioning), the number of CS-US pairings, the contiguity between the CS and US, the strength of the statistical contingency between the presence of the CS and US, changes in the CS-US relations (e.g., extinction, US postexposure, CS preexposure, US preexposure, counterconditioning, US revaluation), whether a CS and US are paired directly or indirectly (i.e., sensory preconditioning, higher order conditioning), whether information about the CS-US relation is presented through experience, observation, or instruction, interactions between different relations (e.g., blocking), and whether the degree of relation between the CS and US is signaled by another stimulus (i.e., occasion setting).

3. If organisms encoded all possible relations between events, a tremendous burden would be imposed on the cognitive system and might 
lead to chaotic behavior (as in the case of schizophrenia; see Carson, Peterson, \& Higgins, 2003). This problem can be managed if propositional encoding needs to occur before a relation can be coded in memory and influence behavior.

4. What is considered to be output depends on the theoretical perspective. For instance, from the perspective of association formation models, one could argue that the output of the association formation process is an expectancy about the occurrence of future events. This perspective leads to the question of whether associative learning effects depend on expectancy awareness (Lovibond \& Shanks, 2002) - that is, awareness of the expectancy that certain future events will occur.

5. The fact that the distal goal to predict significant events has a profound impact on associative learning demonstrates that the process underlying associative learning effects is not goal independent or purely stimulus driven.

6. These elements include not only observable aspects of the external world but also internal, unobservable elements such as knowledge, goals, and dispositions. Internal elements can be assumed to modulate the relation between observable events. For instance, the likelihood that a particular relation in the world leads to a certain change in behavior (i.e., that a particular associative learning effect occurs) can depend on prior knowledge about the additive nature of causes or on the presence of the proximal goal to learn a relation. The assumptions about these internal elements remain functional in nature because they concern only what elements determine behavior, not how the elements determine behavior (e.g., how knowledge is represented or transformed). In terms of the distinction between procedure, effect, and theory that I discussed at the beginning of this article, the functional level of understanding is thus situated at the level of effect, whereas the algorithmic level of understanding is situated at the level of theory.

7. Evaluating stimuli as good or bad could be seen as a good example of an often intuitive and unjustifiable behavior. Studies on evaluative conditioning have shown that evaluations can be changed by pairing stimuli (see De Houwer, Thomas, \& Baeyens, 2001, for a review). Some findings appear to be difficult to reconcile with a propositional account of evaluative conditioning. For instance, evaluative conditioning effects are sometimes unrelated to contingency awareness and goals to detect stimulus contingencies (e.g., Baeyens et al., 1990). However, there are also findings showing a close link between contingency awareness and evaluative conditioning (e.g., Pleyers, Corneille, Luminet, \& Yzerbyt, 2007). At present, it is therefore too early to determine whether (in some cases; see De Houwer, 2007) evaluative conditioning is based on nonpropositional processes.

8. Again, it should be pointed out that evidence against this hypothesis should not necessarily be interpreted as evidence in support of an association formation account. For instance, the automatic activation of memory traces can probably influence behavior in a direct manner- that is, in a way not mediated by propositions. As a result, a stimulus similar to one aspect of a prior experience can result in the activation of the entire memory trace, including representations of other stimuli. If this leads to a change in behavior, it can be called an associative learning effect, because the change is due to a prior experience, or the memory thereof, in which two stimuli co-occurred. Such effects depend on binding processes necessary to create coherent perceptual experiences and memory traces (e.g., Meiser, Sattler, \& Weisser, 2008). Although one could argue that these binding processes are associative in nature, their only function is to create coherent representations of specific events at a specific point in time. Association formation, on the other hand, is typically considered to capture regularities across many separate experiences. Nevertheless, it would be interesting to examine whether associative learning can be based on memory activation only (and thus binding), under which conditions such effects can be observed, and how they differ from associative learning effects based on the generation and evaluations of propositions.

(Manuscript received February 4, 2008; accepted for publication March 2, 2008.) 